\title{
Caracterização clínica, farmacoterapêutica e nutricional de pacientes com Diabetes
}

\section{Mellitus}

\author{
Clinical, pharmacotherapeutic and nutritional characterization of patients with Diabetes Mellitus \\ Caracterización clínica, farmacoterapéutica y nutricional de pacientes con Diabetes Mellitus
}

Recebido: 26/05/2021 | Revisado: 31/05/2021 | Aceito: 04/06/2021 | Publicado: 04/07/2021

Luciano Costa Corsini

ORCID: https://orcid.org/0000-0002-7624-7646 José do Rosário Vellano University, Brazil E-mail: luthfarma2013@gmail.com

André Luiz Oliveira de Faria

ORCID: https://orcid.org/0000-0001-6816-8262 José do Rosário Vellano University, Brazil E-mail: andrelofaria@hotmail.com

Bruno Lacerda Esteves

ORCID: https://orcid.org/0000-0002-2878-6055 José do Rosário Vellano University, Brazil E-mail: brunolacerdaesteves@gmail.com

Bruno Luis Silva

ORCID: https://orcid.org/0000-0001-7245-6670 José do Rosário Vellano University, Brazil E-mail: bruno-loa@hotmail.com

Roberta Bessa Veloso Silva ORCID: https://orcid.org/0000-0003-4794-5872 José do Rosário Vellano University, Brazil E-mail: bessaveloso@yahoo.com.br

Gérsika Bitencourt Santos

ORCID: https://orcid.org/0000-0003-0849-2786 José do Rosário Vellano University, Brazil E-mail: gersika.santos@unifenas.br

\begin{abstract}
Resumo
O diabetes é uma doença de base genética e hereditária, considerada como uma síndrome do metabolismo que resulta no acúmulo de glicose pelo organismo. O tratamento do diabetes tem como intuito prevenir e curar possíveis complicações e manter a qualidade de vida. O objetivo deste estudo é caracterizar os pacientes com diabetes mellitus quanto aos fatores nutricionais, clínicos, à farmacoterapia utilizada. Foi escolhido público alvo de pacientes acima de 15 anos, diagnosticados com diabetes tipo I e II. Foi aplicado um questionário para identificar as variáveis relacionadas ao tipo de tratamento realizado pelo paciente (medicamentoso e não medicamentoso). Além disso foram analisadas a faixa etária, renda, escolaridade, estado civil e variáveis clínicas (tempo de diagnóstico, comorbidades, complicações crônicas), antropométricas, adesão ao tratamento medicamentoso e Frequência de Consumo Alimentar. Neste estudo encontrou-se maior número de pacientes mulheres, com faixa etária maior de 60 anos. Um número elevado de pacientes relatou inatividade física, levando assim uma ampliação do sedentarismo e da circunferência abdominal. A população amostral não faz total adesão aos métodos preconizados para controle do diabetes, pois a maioria dos pacientes entrevistados não fazem devido acompanhamento nutricional e não possuem o hábito de exercícios físicos regulares. Soma-se a isso, uma parte da população estudada que não é aderente ao tratamento medicamentoso.
\end{abstract}

Palavras-chave: Diabetes mellitus; Conduta do tratamento medicamentoso; Dieta saudável.

\begin{abstract}
Diabetes is a genetic and hereditary disease, considered a syndrome of metabolism that results in the accumulation of glucose by the body. Diabetes treatment aims to prevent and cure possible complications and maintain quality of life. The aim of this study is to characterize patients with diabetes mellitus regarding nutritional and clinical factors, the pharmacotherapy used. A target audience of patients over 15 years old, diagnosed with type I and II diabetes was chosen. A questionnaire was applied to identify the variables related to the type of treatment performed by the patient (medicated and non-medicated). In addition, the age group, income, education, marital status and clinical variables (time of diagnosis, comorbidities, chronic complications), anthropometric, adherence to drug treatment and Frequency of Food Consumption were analyzed. This study found a greater number of female patients, aged over 60 years. A high number
\end{abstract}


of patients reported physical inactivity, thus leading to an increase in sedentary lifestyle and waist circumference. The sample population does not fully adhere to the recommended methods for diabetes control, as most of the patients interviewed do not do due nutritional monitoring and do not have the habit of regular physical exercise. In addition, part of the studied population is not adherent to drug treatment.

Keywords: Diabetes mellitus; Drug treatment conduct; Healthy diet.

\section{Resumen}

La diabetes es una enfermedad genética y hereditaria, considerada un síndrome del metabolismo que resulta en la acumulación de glucosa por parte del organismo. El tratamiento de la diabetes tiene como objetivo prevenir y curar posibles complicaciones y mantener la calidad de vida. El objetivo de este estudio es caracterizar a los pacientes con diabetes mellitus en función de los factores nutricionales y clínicos, la farmacoterapia utilizada. Se eligió un público objetivo de pacientes mayores de 15 años, diagnosticados con diabetes tipo I y II. Se aplicó un cuestionario para identificar las variables relacionadas con el tipo de tratamiento realizado por el paciente (medicado y no medicado). Además, se analizaron edad, ingresos, educación, estado civil y variables clínicas (tiempo de diagnóstico, comorbilidades, complicaciones crónicas), antropométricas, adherencia al tratamiento farmacológico y Frecuencia de Consumo de Alimentos. Este estudio encontró un mayor número de pacientes del sexo femenino, mayores de 60 años. Un gran número de pacientes informaron de inactividad física, lo que llevó a un aumento del estilo de vida sedentario y de la circunferencia de la cintura. La población de la muestra no se adhiere plenamente a los métodos recomendados para el control de la diabetes, ya que la mayoría de los pacientes entrevistados no realizan el debido seguimiento nutricional y no tienen el hábito de realizar ejercicio físico regular. Además, parte de la población estudiada no es adherente al tratamiento farmacológico.

Palabras clave: Diabetes mellitus; Conducta de tratamiento farmacológico; Dieta saludable.

\section{Introdução}

Segundo a Sociedade Brasileira de Diabetes (2020), o diabetes é uma doença de base genética e hereditária. É considerada uma síndrome do metabolismo que resulta no acúmulo de glicose pelo organismo. Pacientes com diabetes apresentam deficiência na função da insulina, o hormônio responsável por metabolizar a glicose, ou mesmo apresentam falta de insulina no organismo.

O diabetes mellitus do tipo II tem importante causa de morbidade, incapacidade e mortalidade, sendo este último devido principalmente ao infarto agudo do miocárdio e doença cerebrovascular (DCV), como o paciente diabético é mais propenso a sofrer-lhes porque o fator aterosclerótico associado a hiperglicemia (Nascimento, et al, 2020).

Esta síndrome metabólica é resultado, em parte, a uma tradição de maus hábitos alimentares - alimentação desequilibrada, rica em gorduras, carboidratos, açucares e produtos industrializados e, ao mesmo tempo, pobre em vegetais, legumes e frutas. Soma-se a isso também, o estilo de vida sedentário que quando somado a fatores de riscos como obesidade e tabagismo, contribuem para o aparecimento da doença (Borges, et al, 2021).

$\mathrm{O}$ tratamento do diabetes tem como intuito prevenir e curar possíveis complicações e manter a qualidade de vida. Traçar metas e planos de tratamentos que devem ser criados para os pacientes com base em suas preferências, valores e metas individuais. Várias estratégias e técnicas dietéticas devem ser usadas para apoiar os esforços de autogestão dos pacientes, incluindo o fornecimento de uma educação alimentar sobre habilidades de resolução de problemas para mudanças no estilo de vida em aspectos do cuidado com o diabetes (Assalim, 2018).

Os cuidados com o diabetes devem ser gerenciados por uma equipe multidisciplinar que pode contar com médicos da atenção básica, médicos especializados, enfermeiros, assistentes médicos, enfermeiros, nutricionistas, especialistas em exercícios, farmacêuticos, dentistas e profissionais de saúde mental (Associação Americana De Diabetes, 2019).

Este estudo tem como objetivo caracterizar os pacientes com diabetes mellitus quanto aos fatores sociodemográficos, clínicos, à farmacoterapia utilizada, as medidas antropométricas e o perfil alimentar de tais indivíduos. 


\section{Metodologia}

Este é um estudo transversal realizado em ambulatórios da cidade de Alfenas-MG. A amostra foi constituída por indivíduos portadores de diabetes mellitus atendidos em tais ambulatórios. O trabalho foi aprovado pelo comitê de ética e pesquisa em seres humanos (CEP) da UNIFENAS-Alfenas sob parecer número 3.735.994.

Os critérios de inclusão foram idade maior ou igual a 15 anos, em uso de terapia com antidiabéticos, capacidade cognitiva e auditiva preservadas, em seguimento regular nas instituições selecionadas para o estudo. Foram excluídas da pesquisa as mulheres com diagnóstico de diabetes gestacional.

Os instrumentos de coleta de dados continham informações relacionadas às variáveis sociodemográficas (sexo, idade, escolaridade e renda familiar), clínicas (tempo de diagnóstico, comorbidades, complicações crônicas), antropométricas (Índice de Massa Corpórea - IMC, circunferência abdominal), adesão ao tratamento medicamentoso e Frequência de Consumo Alimentar.

A coleta de dados foi realizada entre fevereiro e maio de 2021 por pesquisadores previamente treinados. Os dados coletados foram organizados e apresentados através de tabelas obtendo-se as frequências absoluta, percentual e intervalos de confiança para proporção com $95 \%$ de confiança. As correlações entre as variáveis foram checadas pelo teste de independência de qui-quadrado, ao nível nominal de 5\% de significância e, nos casos em que as frequências absolutas das tabelas de contingências foram menores ou iguais a que 5, utilizou-se o teste exato de Fisher, (Bussab \& Morettin, 2017).

A análise estatística foi realizada no software $\mathrm{R}^{\circledR}$ (R CORE TEAM, 2021).

\section{Resultados e Discussão}

Os resultados apresentados na Tabela 1 mostram que, na amostra de 50 participantes, observou-se uma frequência 2,2 vezes maior de mulheres do que homens, sendo 34 (68,00\%) mulheres e, 16 (32,00\%), homens. A faixa etária predominante foi de 60 anos ou mais, 24 (48,00\%), seguida de 41 a 59 anos, 14 (28,00\%), e de 0 a 40 anos, 12 (24,00\%). Os participantes têm principalmente ensino fundamental e ensino superior, sendo, 17 (34,00\%) e 17 (34,00\%), respectivamente, e 16 (32,00\%), ensino médio. A renda familiar foi de até 2 salários mínimos, 19 (38,00\%), seguido de 17 (34,00\%), acima de 6 salários mínimos e de 3 a 5 salários mínimos, $14(28,00 \%)$. 
Tabela 1 - Caracterização da amostra, segundo o gênero, estado civil, faixa etária, escolaridade e renda familiar.

\begin{tabular}{|c|c|c|c|}
\hline Variáveis & $n(\%)$ & IC $(p ; 95 \%)$ & Valor-p \\
\hline \multicolumn{4}{|l|}{ Gênero } \\
\hline Feminino & $34(68,00 \%)$ & 53,$17 ; 80,07$ & $0,0162^{*}$ \\
\hline Masculino & $16(32,00 \%)$ & 19,$93 ; 46,83$ & $0,0162^{*}$ \\
\hline Total & $50(100,00 \%)$ & & \\
\hline \multicolumn{4}{|l|}{ Estado civil } \\
\hline Solteiro(a) & $21(42,00 \%)$ & 28,$49 ; 56,73$ & $0,3222 n s$ \\
\hline Casado(a) & $21(42,00 \%)$ & 28,$49 ; 56,73$ & $0,3222 n s$ \\
\hline Viúvo(a) & $8(16,00 \%)$ & 7,$64 ; 29,66$ & $<0,01^{* *}$ \\
\hline Total & $50(100,00 \%)$ & & \\
\hline \multicolumn{4}{|l|}{ Faixa etária } \\
\hline 0 a 40 anos & $12(24,00 \%)$ & 13,$52 ; 38,49$ & $0,0004^{* *}$ \\
\hline 41 a 59 anos & $14(28,00 \%)$ & 16,$67 ; 42,71$ & $0,0030^{* *}$ \\
\hline 60 anos ou mais & $24(48,00 \%)$ & 33,$88 ; 6242$ & $0,8875 n s$ \\
\hline Total & $50(100,00 \%)$ & & \\
\hline \multicolumn{4}{|l|}{ Escolaridade } \\
\hline Ensino Fundamental & $17(34,00 \%)$ & 21,$59 ; 48,85$ & $0,0339^{*}$ \\
\hline Ensino Médio & $16(32,00 \%)$ & 19,$93 ; 46,83$ & $0,0162^{*}$ \\
\hline Ensino Superior & $17(34,00 \%)$ & 21,$59 ; 48,85$ & $0,0339^{*}$ \\
\hline Total & $50(100,00 \%)$ & & \\
\hline \multicolumn{4}{|l|}{ Renda familiar } \\
\hline Até 2 salários mínimos & $19(38,00 \%)$ & 25,$00 ; 52,84$ & $0,1198 n s$ \\
\hline 3 a 5 salários mínimos & $14(28,00 \%)$ & 16,$67 ; 42,71$ & $0,0030^{* *}$ \\
\hline Acima de 6 salários mínimos & $17(34,00 \%)$ & 21,$59 ; 48,85$ & $0,0339^{*}$ \\
\hline Total & $50(100,00 \%)$ & & \\
\hline
\end{tabular}

* Significativo ao nível nominal de $5 \%$ de significância, $(\mathrm{p}<0,05)$.

** Significativo ao nível nominal de $1 \%$ de significância, $(\mathrm{p}<0,01)$.

${ }^{n s}$ Não significativo ao nível nominal de $5 \%$ de significância, $(\mathrm{p}>0,05)$.

Fonte: Autores.

Estudos obtiveram resultados semelhantes ao presente estudo, visto que foi observada uma prevalência maior de mulheres frente ao número de homens na população amostral. Ademais, há uma concordância nos dados referentes a faixa etária e renda familiar, uma vez que nesse estudo utilizado como base houve predomínio de uma população com idade de 60 anos ou mais e com renda de até 2 salários mínimos. Em contrapartida, a discrepância foi mínima no quesito escolaridade e estado civil (Salin, et al, 2019).

Outro estudo observou uma ocorrência maior do sexo feminino em relação ao masculino quanto ao número de pacientes diabéticos. Além do mais, obteve-se incidência maior do diabetes mellitus na faixa etária de 60 ou mais anos já que nessa idade a população tende a diminuir a prática de exercícios físicos e a manutenção de uma dieta controlada. Sendo assim, resulta, muitas vezes, em um aumento da circunferência abdominal, do braço, da cintura e do quadril e, quando se tem a soma de todos esses fatores pode acarretar em uma obesidade, a qual é um dos principais fatores de risco para o aparecimento do diabetes (Almeida Roediger, et al, 2016).

Em se tratando da avaliação antropométrica, na Tabela 2, verifica-se que $22(44,00 \%)$ entrevistados, têm de $21 \mathrm{~cm}$ a 30 $\mathrm{cm}$ de circunferência do braço; $18(36,00 \%), 31 \mathrm{~cm}$ ou mais e $10(20,00 \%)$, apresentam a circunferência do braço ente15 $\mathrm{cm}$ e $20 \mathrm{~cm}$. Dos 50 participantes, $26(52,00 \%)$, apresentaram circunferência do quadril de $50 \mathrm{~cm}$ a $100 \mathrm{~cm} ; 22(44,00 \%)$ de $101 \mathrm{~cm}$ a $120 \mathrm{~cm}$ e $2(4,00 \%)$, de $121 \mathrm{~cm}$ ou mais. Quanto à circunferência da coxa, 23 (46,00\%), mediram de $31 \mathrm{~cm}$ a $60 \mathrm{~cm} ; 15(30,00 \%)$, de $15 \mathrm{~cm}$ a $20 \mathrm{~cm}$ e $12(24,00 \%)$, de $21 \mathrm{~cm}$ a $30 \mathrm{~cm}$. O tamanho da circunferência da cintura em 30 (60,00\%) dos participantes foi de $101 \mathrm{~cm}$ a $120 \mathrm{~cm}$, seguido de $50 \mathrm{~cm}$ a $100 \mathrm{~cm}, 18(36,00 \%)$, e $121 \mathrm{~cm}$ ou acima, 2(4,00\%). 
Pode-se observar, na Tabela 2, que a frequência de participantes com sobrepeso foi 1,8 vezes maior do que àqueles que apresentaram obesidade tipo I e, 2,2 vezes maior do que os que eutrofia, sendo, respectivamente, 24 (48,00\%), 13 (26,00\%), 11 $(22,00 \%)$ e, obesidade tipo II, 2 (4,00\%). Apesar de a grande maioria estar com sobrepeso, dos 57 hábitos identificados (pelo menos um deles), 23 (40,35\%), relataram a prática de atividade física e 17 (29,82\%), fazem acompanhamento nutricional. Em contrapartida, 15 (26,32\%), têm o hábito de ingerir álcool e 2 (3,51\%) são fumantes. Verificou-se que dos 50 participantes, 32 $(64,00 \%)$ apresentaram risco para desenvolver doença coronariana.

Tabela 2 - Variáveis referentes à avaliação antropométrica, IMC, hábitos de vida e o risco de desenvolver doença coronariana.

\begin{tabular}{|c|c|c|c|c|}
\hline Variáveis & $n(\%)$ & & IC $(p ; 95 \%)$ & Valor-p \\
\hline & \multicolumn{4}{|c|}{ Avaliação antropométrica } \\
\hline \multicolumn{5}{|l|}{ Circunferência do braço } \\
\hline $15 \mathrm{~cm}$ a $20 \mathrm{~cm}$ & $10(20,00 \%)$ & & 10,$50 ; 34,14$ & $<0,01^{* *}$ \\
\hline $21 \mathrm{~cm}$ a $30 \mathrm{~cm}$ & $22(44,00 \%)$ & & 30,$27 ; 58,65$ & $0,4795 n s$ \\
\hline $31 \mathrm{~cm}$ ou acima & $18(36,00 \%)$ & & 23,$28 ; 58,86$ & $0,0660 \mathrm{~ns}$ \\
\hline Total & $50(100,00 \%)$ & & & \\
\hline \multicolumn{5}{|l|}{ Circunferência do quadril } \\
\hline $50 \mathrm{~cm}$ a $100 \mathrm{~cm}$ & $26(52,00 \%)$ & & 37,$57 ; 66,12$ & $0,8875 n s$ \\
\hline $101 \mathrm{~cm}$ a $120 \mathrm{~cm}$ & $22(44,00 \%)$ & & 30,$27 ; 58,65$ & $0,4795 n s$ \\
\hline $121 \mathrm{~cm}$ ou acima & $2(4,00 \%)$ & & 0,$69 ; 14,86$ & $<0,01^{* *}$ \\
\hline Total & $50(100,00 \%)$ & & & \\
\hline \multicolumn{5}{|l|}{ Circunferência da coxa } \\
\hline $15 \mathrm{~cm}$ a $20 \mathrm{~cm}$ & $15(30,00 \%)$ & & 18,$28 ; 44,78$ & $0,0072^{* *}$ \\
\hline $21 \mathrm{~cm}$ a $30 \mathrm{~cm}$ & $12(24,00 \%)$ & & 13,$52 ; 39,49$ & $0,0004^{* *}$ \\
\hline $31 \mathrm{cma} 60 \mathrm{~cm}$ & $23(46,00 \%)$ & & 32,$06 ; 60,55$ & $0,6714 n s$ \\
\hline Total & $50(100,00 \%)$ & & & \\
\hline \multicolumn{5}{|l|}{ Circunferência da cintura } \\
\hline $50 \mathrm{~cm}$ a $100 \mathrm{~cm}$ & $18(36,00 \%)$ & & 23,$28 ; 58,86$ & $0,0660 n s$ \\
\hline $101 \mathrm{~cm}$ a $120 \mathrm{~cm}$ & $30(60,00 \%)$ & & 45,$20 ; 73,27$ & $0,2031 n s$ \\
\hline $121 \mathrm{~cm}$ ou acima & $2(4,00 \%)$ & & 0,$69 ; 14,86$ & $<0,01^{* *}$ \\
\hline Total & $50(100,00 \%)$ & & & \\
\hline Eutrofia & IMC & & & \\
\hline $18,5 \mathrm{~kg} / \mathrm{m}^{2}$ a $24,5 \mathrm{~kg} / \mathrm{m}^{2}$ & $11(22,00 \%)$ & & 11,$99 ; 36,33$ & $0,0001^{* *}$ \\
\hline \multicolumn{5}{|l|}{ Sobrepeso } \\
\hline $25,0 \mathrm{~kg} / \mathrm{m}^{2}$ a $29,9 \mathrm{~kg} / \mathrm{m}^{2}$ & $24(48,00 \%)$ & & 33,$88 ; 62,42$ & $0,8875 n s$ \\
\hline \multicolumn{5}{|l|}{ Obesidade tipo 1} \\
\hline $30,0 \mathrm{~kg} / \mathrm{m}^{2}$ a $34,9 \mathrm{~kg} / \mathrm{m}^{2}$ & $13(26,00 \%)$ & & 15,$08 ; 40,61$ & $0,0011^{* *}$ \\
\hline \multicolumn{5}{|l|}{ Obesidade tipo 2} \\
\hline $35,0 \mathrm{~kg} / \mathrm{m}^{2}$ a $39,9 \mathrm{~kg} / \mathrm{m}^{2}$ & $2(4,00 \%)$ & & 0,$69 ; 14,86$ & $<0,01^{* *}$ \\
\hline \multirow[t]{3}{*}{ Total } & $50(100,00 \%)$ & & & \\
\hline & Hábitos de vid & & & \\
\hline & Sim & Não & & \\
\hline Prática de atividade física & $23(40,35 \%)$ & $27(18,88 \%)$ & 5,$98 ; 36,96$ & $0,0028^{* *}$ \\
\hline Ingestão de álcool & $15(26,32 \%)$ & $35(24,47 \%)$ & 12,$81 ; 16,49$ & $0,9279 n s$ \\
\hline Fumante & $2(3,51 \%)$ & $48(33,57 \%)$ & 19,$74 ; 40,38$ & $<0,01^{* *}$ \\
\hline Acompanhamento nutricional & $17(29,82 \%)$ & $33(23,08 \%)$ & 8,$21 ; 21,71$ & $0,4157 n s$ \\
\hline \multirow[t]{2}{*}{ Total } & $57(100,00 \%)$ & $143(100,00 \%)$ & & \\
\hline & \multicolumn{4}{|c|}{ Risco de desenvolver doença coronariana } \\
\hline Sim & $32(64,00 \%)$ & & 49,$14 ; 76,71$ & $0,0660 \mathrm{~ns}$ \\
\hline Não & $18(36,00 \%)$ & & 23,$28 ; 50,86$ & $0,0660 \mathrm{~ns}$ \\
\hline Total & $50(100,00 \%)$ & & & \\
\hline
\end{tabular}

* Significativo ao nível nominal de 5\% de significância, $(\mathrm{p}<0,05)$.

** Significativo ao nível nominal de $1 \%$ de significância, $(\mathrm{p}<0,01)$.

${ }^{n s}$ Não significativo ao nível nominal de $5 \%$ de significância, (p>0,05).

Fonte: Autores. 
Devido ao grupo de estudo ser predominantemente representado por pessoas com sobrepeso, obesidade I e II, esses pacientes apresentam majoritariamente a circunferência abdominal, do braço, da coxa e do quadril mais elevados que os valores de referência. Sendo assim, nota-se o visível risco do desenvolvimento de ocorrências cardiológicas, principalmente, a doença arterial coronariana (Barroso, et al, 2017).

O cruzamento entre os estudos relacionados ao IMC, indica uma alta ocorrência entre o diferencial de gorduras, do índice da massa corporal, do IMC de sobrepeso e da obesidade tipo 1. Em decorrência a estes resultados, o sobrepeso mostra uma mínima elevação da gordura corporal, em seguida é possível analisar a obesidade grau 1, que apresenta um alto índice da gordura corporal (Santos, et al, 2020).

Na Tabela 3, dentre as patologias levantadas, àquelas que ocorreram com maiores frequências foram, nesta ordem, diabetes mellitus, 50 (28,74\%); hipertensão arterial, 28 (16,09\%); depressão ou ansiedade, 22 (12,64\%); dores na coluna, 22 (12,64\%); hipercolesterolemia, 17 (9,77\%); doenças cardíacas, 10 (5,75\%); artrite, 10 (5,75\%); doença renal crônica, 6 (3,45\%), câncer, 6 (3,45\%); bronquite/asma, 3 (1,72\%) e acidente vascular cerebral, 2 (1,15\%).Dos 50 participantes, 37 (74,00\%), apresentaram diabetes mellitus tipo II, ao passo que 13 (26,00\%), diabetes mellitus tipo I. O tempo de diagnóstico da doença foi para $28(56,00 \%)$, superior a 10 anos; $14(28,00 \%)$, de 3 a 10 anos e $8(16,00 \%)$, de 0 a 2 anos. O valor observado da glicemia foi 121 a $200 \mathrm{mg} / \mathrm{dl}$ para $24(48,00 \%)$, seguido de $14(28,00 \%), 201 \mathrm{mg} / \mathrm{dl}$ ou acima e $12(24,00 \%)$, de 100 a $120 \mathrm{mg} / \mathrm{dl}$.

Dentre os antidiabéticos injetáveis, a insulina regular e a insulina NPH foram as mais usadas, sendo 13 (44,83\%)e 6 (20,69\%), respectivamente. Verificou-se o uso de insulina Aspart e a insulina Glargina em $4(13,79 \%)$ e $4(13,79 \%)$ dos participantes, respectivamente, $1(3,45 \%)$ paciente utiliza a insulina Glulisina e $1(3,45 \%)$ faz uso da insulina Degludeca + Liraglutida. Dos antidiabéticos orais, 16 (51,61\%) entrevistados utilizam a Metformina XR; 10 (32,26\%), Metformina e 3 (9,68\%), a Glicazida. Em menor frequência, a Dapaglifozina, 1 (3,22\%) e a Alogliptina, 1 (3,22\%), (TABELA 3).

Em relação à adesão ao tratamento, dos 43 (21,50\%), que responderam “sim”, 16 (37,21\%), relataram que esquecem alguma vez de tomar o remédio; 13 (30,23\%), afirmaram descuido com os horários dos remédios; 9 (20,93\%), declararam que param de tomar quando se sentem mal e, 5 (11,63\%), param de tomar quando se sentem bem. 
Tabela 3 - Variáveis referentes às patologias, tipo de diabetes, tempo de diagnóstico da doença, valor da glicemia, adesão ao tratamento, entre outras.

\begin{tabular}{|c|c|c|c|c|}
\hline Variáveis & $n(\%)$ & & IC $(p ; 95 \%)$ & Valor-p \\
\hline & Patologias & & & \\
\hline Hipertensão arterial & $28(16,09 \%)$ & & 11,$13 ; 22,59$ & $<0,01^{* *}$ \\
\hline Hipercolesterolemia & $17(9,77 \%)$ & & 5,$96 ; 15,42$ & $<0,01^{* *}$ \\
\hline Doenças cardíacas & $10(5,75 \%)$ & & 2,$95 ; 10,61$ & $<0,01^{* *}$ \\
\hline Acidente vascular cerebral & $2(1,15 \%)$ & & 0,$20 ; 4,52$ & $<0,01^{* *}$ \\
\hline Diabetes mellitus & $50(28,74 \%)$ & & 22,$27 ; 36,16$ & $<0,01^{* *}$ \\
\hline Depressão ou ansiedade & $22(12,64 \%)$ & & 8,$26 ; 18,73$ & $<0,01^{* *}$ \\
\hline Dores na coluna & $22(12,64 \%)$ & & 8,$26 ; 18,73$ & $<0,01^{* *}$ \\
\hline Artrite & $10(5,75 \%)$ & & 2,$95 ; 10,61$ & $<0,01^{* *}$ \\
\hline Bronquite/Asma & $3(1,72 \%)$ & & 0,$45 ; 5,36$ & $<0,01^{* *}$ \\
\hline Doença renal crônica & $6(3,45 \%)$ & & 1,$41 ; 7,69$ & $<0,01^{* *}$ \\
\hline Câncer & $4(2,30 \%)$ & & 0,$74 ; 6,16$ & $<0,01^{* *}$ \\
\hline \multirow[t]{2}{*}{ Total } & $174(100,00 \%)$ & & & \\
\hline & Tipo de diabetes & & & \\
\hline Tipo I & $13(26,00 \%)$ & & 15,$08 ; 40,61$ & $0,0011^{* *}$ \\
\hline Tipo II & $37(74,00 \%)$ & & 59,$39 ; 84,92$ & $0,0011^{* *}$ \\
\hline \multirow[t]{2}{*}{ Total } & $50(100,00 \%)$ & & & \\
\hline & Tempo de diagnós & da doença & & \\
\hline 0 a 2 anos & $8(16,00 \%)$ & & 7,$64 ; 29,66$ & $<0,01^{* *}$ \\
\hline 3 a 10 anos & $14(28,00 \%)$ & & 16,$67 ; 42,71$ & $0,0030^{* *}$ \\
\hline 11 anos ou acima & $28(56,00 \%)$ & & 41,$65 ; 69,73$ & $0,4795 n s$ \\
\hline \multirow[t]{2}{*}{ Total } & $50(100,00 \%)$ & & & \\
\hline & Valor da glicemia & & & \\
\hline 100 a $120 \mathrm{mg} / \mathrm{dl}$ & $12(24,00 \%)$ & & 13,$52 ; 39,49$ & $0,0004^{* *}$ \\
\hline 121 a $200 \mathrm{mg} / \mathrm{dl}$ & $24(48,00 \%)$ & & 33,$88 ; 62,42$ & $0,8875 n s$ \\
\hline $201 \mathrm{mg} / \mathrm{dlou}$ acima & $14(28,00 \%)$ & & 16,$67 ; 42,71$ & $0,0030^{* *}$ \\
\hline Total & $50(100,00 \%)$ & & & \\
\hline \multicolumn{5}{|l|}{ Antidiabéticos injetáveis } \\
\hline Insulina Regular & $13(44,83 \%)$ & & 26,$95 ; 64,02$ & $0,7103 n s$ \\
\hline Insulina NPH & $6(20,69 \%)$ & & 8,$70 ; 40,26$ & $0,0030^{* *}$ \\
\hline Insulina Glulisina & $1(3,45 \%)$ & & 0,$18 ; 19,63$ & $<0,01^{* *}$ \\
\hline Insulina Aspart & $4(13,79 \%)$ & & 4,$51 ; 32,57$ & $0,0002^{* *}$ \\
\hline Insulina Glargina & $4(13,79 \%)$ & & 4,$51 ; 32,57$ & $0,0002^{* *}$ \\
\hline Insulina Degludeca + Liraglutida & $1(3,45 \%)$ & & 0,$18 ; 19,63$ & $<0,01^{* *}$ \\
\hline Total & $29(100,00 \%)$ & & & \\
\hline \multicolumn{5}{|l|}{ Antidiabéticos orais } \\
\hline Metformina & $10(32,26 \%)$ & & 17,$32 ; 51,46$ & $0,0725 \mathrm{~ns}$ \\
\hline Metformina XR & $16(51,61 \%)$ & & 33,$40 ; 69,44$ & $1,0000 n s$ \\
\hline Glicazida & $3(9,68 \%)$ & & 2,$53 ; 26,90$ & $<0,01^{* *}$ \\
\hline Dapaglifozina & $1(3,22 \%)$ & & 0,$17 ; 18,51$ & $<0,01^{* *}$ \\
\hline Alogliptina & $1(3,22 \%)$ & & 0,$17 ; 18,51$ & $<0,01^{* *}$ \\
\hline Total & $31(100,00 \%)$ & & & \\
\hline Adesão ao tratamento & Sim & Não & & \\
\hline $\begin{array}{l}\text { Esquece alguma vez de tomar o } \\
\text { remédio }\end{array}$ & $16(37,21 \%)$ & $34(21,66 \%)$ & 1,$74 ; 32,85$ & $0,0590 \mathrm{~ns}$ \\
\hline $\begin{array}{l}\text { Descuido com os horários da } \\
\text { medicação }\end{array}$ & $13(30,23 \%)$ & $37(23,57 \%)$ & 10,$06 ; 23,39$ & $0,4867 n s$ \\
\hline Para de tomar (caso sinta bem) & $5(11,63 \%)$ & $45(28,66 \%)$ & 3,$64 ; 30,42$ & $0,0369^{*}$ \\
\hline Para de tomar (caso sinta mal) & $9(20,93 \%)$ & $41(26,11 \%)$ & 10,$26 ; 20,63$ & $0,6193 n s$ \\
\hline Total & $43(100,00 \%)$ & $157(100,00 \%)$ & & \\
\hline
\end{tabular}

* Significativo ao nível nominal de $5 \%$ de significância, $(\mathrm{p}<0,05)$.

** Significativo ao nível nominal de $1 \%$ de significância, $(\mathrm{p}<0,01)$.

${ }^{n s}$ Não significativo ao nível nominal de $5 \%$ de significância, (p>0,05).

Fonte: Autores. 
Solbiati, et al (2018) mostraram em sua pesquisa, que grande parte do seu grupo amostral não se esquece de tomar remédio e não para de tomar - caso se sinta bem. Dessa forma, há uma concordância com os dados obtidos no presente trabalho, visto que os pacientes entrevistados predominantemente não deixam e nem param de tomar os medicamentos.

Outro estudo mostrou que que os altos valores encontrados referentes ao índice glicêmico são decorrentes de falhas na adesão ao tratamento medicamentoso, sendo ele hipoglicemiante oral ou insulina. Nesse sentido, no presente estudo, foi encontrado um número maior de pacientes que fazem corretamente a adesão ao tratamento frente aos que não fazem. Em contrapartida, os valores glicêmicos colhidos foram de 121-200 MG/DL majoritariamente. Logo, há uma incoerência referente aos tratamentos hipoglicemiantes e as respostas obtidas no questionário de MORISKY (Anjos, et al, 2020).

Em relação ao número de refeições diárias, dos 50 participantes, 39 (78\%), relataram fazer de 2 a 5 refeições e 11 (22\%), de 6 ou mais (resultados não apresentados).

$\mathrm{Na}$ Tabela 4 está apresentada a frequência alimentar diária e ela se baseia em legumes, 42 (7,86\%); frutas, 33 (6,18\%); vegetais folhosos, 32 (5,99\%); bolachas, biscoitos, bolos e pães, 32 (5,99\%); feijão carioca, 40 (7,49\%); óleo de soja, carne bovina, 23 (4,31\%), dentre outros. No consumo semanal destacam-se o peixe, 23 (7,23\%); aves, 20 (6,29\%); frituras, 20 (6,29\%); ovo cozido, 18 (5,66\%); carne suína, 18 (5,66\%); iogurte, 17 (5,34\%); requeijão, 16 (5,03\%); batata, inhame, mandioca, 16 (5,03\%); ovo frito, 15 (4,72\%), e carne bovina, 11 (3,46\%). Identificou-se em 12 (7,79\%) dos participantes, no consumo quinzenal, a presença de embutidos e defumados; lanches e/ou pizzas, 9 (5,84\%); feijão preto, 8 (5,19\%); em 7 (4,54\%), sorvete e/ou açaí; 7 (4,54\%), carne suína; 7 (4,54\%), dentre outras.

Identificou-se em 12 (7,79\%) dos participantes, no consumo quinzenal, a presença de embutidos e defumados; lanches e/ou pizzas, 9 (5,84\%); feijão preto, 8 (5,19\%); em 7 (4,54\%), sorvete e/ou açaí; 7 (4,54\%) e carne suína; 7 (4,54\%), dentre outras. Deve-se destacar na frequência alimentar mensal que 14 (7,61\%) consomem salgadinho, 14 (7,61\%), sorvete e/ou açaí; 12 (6,52\%), embutidos e defumados; 11 (5,98\%), lanches e/ou pizzas; 10 (5,43\%), queijo minas; 10 (5,43\%), lentilha, ervilha, grão de bico ou soja; 9 (4,89\%), carne suína e 9 (4,89\%), suco natural. Em se tratando de nenhum dos alimentos consumidos, verificou-se o leite zero lactose, 48 (10,46\%); leite em pó, 41 (8,93\%); leite desnatado, 33 (7,19\%); arroz integral, 32 (6,97\%); leite integral, 30 (6,54\%); feijão preto, 32 (6,97\%); salgadinho, 21 (4,57\%); suco industrializado, 21 (4,57\%) e lanches e/ou pizzas, $20(4,36 \%)$, (Tabela 4). 
Tabela 4 - Variáveis referentes à alimentação diária, semanal, quinzenal, mensal e nenhuma frequência de consumo dos alimentos considerados.

\begin{tabular}{|c|c|c|c|c|c|}
\hline Variáveis & $n(\%)$ & & & & \\
\hline Frequência alimentar & Diária & Semanal & Quinzenal & Mensal & Nenhum \\
\hline Frutas & $33(6,18 \%)$ & $8(2,52 \%)$ & $6(3,90 \%)$ & $3(1,63 \%)$ & $0(0,00 \%)$ \\
\hline Legumes & $42(7,86 \%)$ & $3(0,94 \%)$ & $3(1,95 \%)$ & $1(0,54 \%)$ & $1(0,22 \%)$ \\
\hline Vegetais folhosos & $32(5,99 \%)$ & $11(3,46 \%)$ & $3(1,95 \%)$ & $2(1,09 \%)$ & $2(0,43 \%)$ \\
\hline Saladas cruas & $29(5,43 \%)$ & $11(3,46 \%)$ & $2(1,30 \%)$ & $5(2,72 \%)$ & $3(0,65 \%)$ \\
\hline Ovo frito & $11(2,06 \%)$ & $15(4,72 \%)$ & $6(3,90 \%)$ & $7(3,80 \%)$ & $11(2,39 \%)$ \\
\hline Ovo cozido & $11(2,06 \%)$ & $18(5,66 \%)$ & $3(1,95 \%)$ & $5(2,72 \%)$ & $13(2,83 \%)$ \\
\hline Aves & $17(3,18 \%)$ & $20(6,29 \%)$ & $6(3,90 \%)$ & $3(1,63 \%)$ & $4(0,87 \%)$ \\
\hline Peixes & $9(1,68 \%)$ & $23(7,23 \%)$ & $6(3,90 \%)$ & $2(1,09 \%)$ & $10(2,18 \%)$ \\
\hline Carne bovina & $23(4,31 \%)$ & $11(3,46 \%)$ & $5(3,25 \%)$ & $6(3,26 \%)$ & $5(1,09 \%)$ \\
\hline Carne suína & $9(1,68 \%)$ & $19(5,97 \%)$ & $7(4,54 \%)$ & $9(4,89 \%)$ & $6(1,31 \%)$ \\
\hline Embutidos e defumados & $7(1,31 \%)$ & $6(1,89 \%)$ & $12(7,79 \%)$ & $12(6,52 \%)$ & $13(2,83 \%)$ \\
\hline Frituras & $5(0,94 \%)$ & $20(6,29 \%)$ & $6(3,90 \%)$ & $7(3,80 \%)$ & $12(2,61 \%)$ \\
\hline $\begin{array}{l}\text { Bolachas, biscoitos, bolos } \\
\text { e pães }\end{array}$ & $32(5,99 \%)$ & $11(3,46 \%)$ & $3(1,95 \%)$ & $2(1,09 \%)$ & $2(0,43 \%)$ \\
\hline Salgadinho & $2(0,37 \%)$ & $7(2,20 \%)$ & $6(3,90 \%)$ & $14(7,61 \%)$ & $21(4,57 \%)$ \\
\hline Sorvete e/ou açaí & $4(0,75 \%)$ & $6(1,89 \%)$ & $7(4,54 \%)$ & $14(7,61 \%)$ & $19(4,14 \%)$ \\
\hline Lanches e/ou pizzas & $2(0,37 \%)$ & $8(2,52 \%)$ & $9(5,84 \%)$ & $11(5,98 \%)$ & $20(4,36 \%)$ \\
\hline Leite desnatado & $7(1,31 \%)$ & $4(1,26 \%)$ & $6(3,90 \%)$ & $0(0,00 \%)$ & $33(7,19 \%)$ \\
\hline Leite integral & $16(3,00 \%)$ & $2(0,63 \%)$ & $1(0,65 \%)$ & $2(1,09 \%)$ & $30(6,54 \%)$ \\
\hline Leite zero lactose & $2(0,37 \%)$ & $0(0,00 \%)$ & $0(0,00 \%)$ & $0(0,00 \%)$ & $48(10,46 \%)$ \\
\hline Leite em pó & $1(0,19 \%)$ & $2(0,63 \%)$ & $2(1,30 \%)$ & $4(2,17 \%)$ & $41(8,93 \%)$ \\
\hline Iogurte & $11(2,06 \%)$ & $17(5,34 \%)$ & $6(3,90 \%)$ & $5(2,72 \%)$ & $11(2,39 \%)$ \\
\hline Queijo minas & $17(3,18 \%)$ & $6(1,89 \%)$ & $6(3,90 \%)$ & $10(5,43 \%)$ & $9(1,96 \%)$ \\
\hline Requeijão & $15(2,81 \%)$ & $16(5,03 \%)$ & $5(3,25 \%)$ & $5(2,72 \%)$ & $9(1,96 \%)$ \\
\hline Arroz branco & $34(6,34 \%)$ & $11(3,46 \%)$ & $0(0,00 \%)$ & $0(0,00 \%)$ & $5(1,09 \%)$ \\
\hline Arroz integral & $7(1,31 \%)$ & $4(1,26 \%)$ & $2(1,30 \%)$ & $5(2,72 \%)$ & $32(6,97 \%)$ \\
\hline Batata, inhame, mandioca & $16(3,00 \%)$ & $16(5,03 \%)$ & $5(3,25 \%)$ & $10(5,43 \%)$ & $3(0,65 \%)$ \\
\hline Feijão preto & $0(0,00 \%)$ & $3(0,94 \%)$ & $8(5,19 \%)$ & $9(4,89 \%)$ & $30(6,54 \%)$ \\
\hline Feijão carioca & $40(7,49 \%)$ & $2(0,63 \%)$ & $2(1,30 \%)$ & $0(0,00 \%)$ & $6(1,31 \%)$ \\
\hline $\begin{array}{l}\text { Lentilha, ervilha, grão de } \\
\text { bico ou soja }\end{array}$ & $16(3,00 \%)$ & $4(1,26 \%)$ & $7(4,54 \%)$ & $10(5,43 \%)$ & $13(2,83 \%)$ \\
\hline Óleo de soja & $31(5,80 \%)$ & $6(1,89 \%)$ & $3(1,95 \%)$ & $0(0,00 \%)$ & $8(1,74 \%)$ \\
\hline Azeite de oliva & $17(3,18 \%)$ & $12(3,77 \%)$ & $4(2,60 \%)$ & $7(3,80 \%)$ & $10(2,18 \%)$ \\
\hline Suco natural & $21(3,93 \%)$ & $9(2,83 \%)$ & $5(3,25 \%)$ & $9(4,89 \%)$ & $6(1,31 \%)$ \\
\hline Suco industrializado & $15(2,81 \%)$ & $7(2,20 \%)$ & $2(1,30 \%)$ & $5(2,72 \%)$ & $21(4,57 \%)$ \\
\hline Total & $534(100,00 \%)$ & $318(100,00 \%)$ & $154(100,00 \%)$ & $184(100,00 \%)$ & $459(100,00 \%)$ \\
\hline
\end{tabular}

Fonte: Autores.

Vargas, et al (2021) concluíram em seu estudo a importância de uma alimentação rica em fibras, triglicerídeos de cadeia média e diminuição de alimentos inflamatórios (como açúcares refinados, lipídeos saturados e alimentos industrializados). Nesse sentido, através desses hábitos relatados fica de fato notório, que o aumento do índice glicêmico é diretamente proporcional aos maus hábitos alimentares.

Outro estudo mostrou que sua população amostral detinha conhecimento satisfatório acerca de hábitos alimentares padronizados para diabéticos. Por outro lado, nesse trabalho foi aferido que mais da metade dos pacientes entrevistados não fazem acompanhamento nutricional. Nessa perspectiva, fica evidente a dificuldade da manutenção de uma alimentação balanceada para o controle glicêmico (Kuczynski, et al 2019). 
Na Tabela 5 estão apresentadas as correlações de interesse, sendo consideradas significativas ao nível nominal de 5\% de significância. O valor-p indica significância estatística para as variáveis faixa etária e o IMC, tipo de diabetes e o IMC; risco de desenvolver doença coronariana e o IMC, acompanhamento nutricional e o valor da glicemia, faixa etária e o tipo de diabetes, circunferência da cintura e o tipo de diabetes, $(\mathrm{p}<0,05)$.

Tabela 5 - Correlações entre as variáveis de interesse.

\begin{tabular}{l|l}
\hline Variáveis & Valor-p \\
\hline Gênero $v s$ IMC & $0,0947 \mathrm{~ns}$ \\
Faixa etária $v s$ IMC & $0,0180^{*}$ \\
Escolaridade $v s$ IMC & $0,1943 \mathrm{~ns}$ \\
Renda familiar $v s$ IMC & $0,6270 \mathrm{~ns}$ \\
Tipo de diabetes $v s$ IMC & $0,0034^{* *}$ \\
Acompanhamento nutricional $v s$ IMC & $0,9374 \mathrm{~ns}$ \\
Risco de desenvolver doença coronariana $v s$ IMC & $<0,01^{* *}$ \\
Esquece de alguma vez tomar a medicação $v s$ valor & $0,3503 \mathrm{~ns}$ \\
da glicemia & \\
Acompanhamento nutricional $v s$ valor da glicemia & $0,0399^{*}$ \\
Gênero $v s$ tipo de diabetes & $1,0000 \mathrm{~ns}$ \\
Faixa etária t $v s$ tipo de diabetes & $<0,01^{* *}$ \\
Valor da glicemia $v s$ tipo de diabetes & $0,7690 \mathrm{~ns}$ \\
Prática de atividade física $v s$ tipo de diabetes & $0,1031 \mathrm{~ns}$ \\
Acompanhamento nutricional $v s$ tipo de diabetes & $0,0987 \mathrm{~ns}$ \\
Número de refeições ao dia $v s$ tipo de diabetes & $0,7044 \mathrm{~ns}$ \\
Circunferência da cintura $v s$ tipo de diabetes & $<0,01^{* *}$ \\
Gênero $v s$ circunferência da cintura & $0,7788 \mathrm{~ns}$ \\
\hline
\end{tabular}

* Significativo ao nível nominal de $5 \%$ de significância, $(\mathrm{p}<0,05)$.

** Significativo ao nível nominal de $1 \%$ de significância, $(\mathrm{p}<0,01)$.

${ }^{n s}$ Não significativo ao nível nominal de $5 \%$ de significância, (p>0,05).

Fonte: Autores.

Em se tratando da relação entre a faixa etária e o IMC, verificou-se que dos 50 participantes, 26 (52,00\%), foram classificados com sobrepeso e, desses, (61,54\%), estão na faixa etária 60 anos ou mais. Ao associar o tipo de diabetes e o IMC, dos $23(46,00 \%)$ com sobrepeso, 18 (78,26\%), apresentaram diabetes tipo II. O risco de desenvolver doença coronariana foi identificado em 16 (72,73\%), dos $22(44,00 \%)$ com sobrepeso.

A correlação entre o acompanhamento nutricional e o valor da glicemia, mostrou que entre os 17 (34,00\%) que relataram que o fazem, $9(52,94 \%)$, apresentaram glicemia de 121 a $200 \mathrm{ml} / \mathrm{dl} ;(41,18 \%)$, de 100 a $120 \mathrm{ml} / \mathrm{dl}$ e $1(5,88 \%), 121 \mathrm{ml} / \mathrm{dl}$ ou mais. Já a relação entre a faixa etária e o tipo de diabetes evidenciou que dos $23(46,00 \%)$ com 60 anos ou mais, 22 (95,65\%), têm o diabetes tipo II; dos $14(28,00 \%)$ com 41 a 59 anos, 13 (92,86\%), também têm o tipo II, porém, dos 13 (26,00\%) na faixa etária de 0 a 40 anos, 11 (84,62\%), apresentaram diabetes tipo I. Outra relação significativa foi entre a circunferência da cintura e o tipo de diabetes. Dos 30 (60,00\%) com circunferência da cintura de 101 a $120 \mathrm{~cm}, 28$ (93,33\%), têm diabetes tipo II e dos 18 $(40,00 \%)$ que mediram entre 50 a $100 \mathrm{~cm}, 11(61,11 \%)$, diabetes tipo I.

\section{Conclusão}

A população amostral não faz total adesão aos métodos preconizados para controle do diabetes mellitus I e II, pois a maioria dos pacientes entrevistados não fazem devido acompanhamento nutricional e não possuem o hábito de exercícios físicos regulares. Soma-se a isso, uma parte da população estudada não é aderente ao tratamento medicamentoso. Por fim, para a melhora 
desse cenário é fundamental a intervenção e trabalho de equipes multiprofissionais, visto que dessa maneira haverá abrangência mais ampla nos tratamentos da diabetes mellitus.

\section{Referências}

Aguiar, C., Duarte, R., \& Carvalho, D. (2019). Nova abordagem para o tratamento da diabetes: da glicemia à doenca cardiovascular. Revista Portuguesa de ( 63 .

Assalim, L., de Carvalho, A. T. P., Barbosa, L. M. P. B., Soto, M. L. M., de Sousa, J. N., Casseb, G. R. M., \& Colucci, R. F. (2018). Dieta Cetogênica no Tratamento de Diabetes Mellitus Tipo 2. International Journal of Nutrology, 11(S 01), Trab304.

Barroso, T. A., Marins, L. B., Alves, R., Gonçalves, A. C. S., Barroso, S. G., \& Rocha, G. D. S. (2017). Associação entre a obesidade central e a incidência de doenças e fatores de risco cardiovascular. International Journal of Cardiovascular Sciences, 30(5), $416-424$.

Borges, A. C. L., \& da Conceição, I. S. P. (2021). Benefícios Dos Alimentos Funcionais No Controle E Tratamento Do Diabetes Mellitus (Dm)-Revisão De Literatura. Hygeia-Revista Brasileira de Geografia Médica e da Saúde, 17, 26-44

Brutti, B., Flores, J., Hermes, J., Martelli, G., da Silva Porto, D., \& Anversa, E. T. R. (2019). Diabete Mellitus: definição, diagnóstico, tratamento e mortalidade no Brasil, Rio Grande do Sul e Santa Maria, no período de 2010 a 2014. Brazilian Journal of Health Review, 2(4), $3174-3182$.

Bussab; P. A.; Morettin, W. de O. Estatística Básica. 9 ed. Editora Saraiva.

da Silva, A. D., Júnior, N. M., Damasceno, D. D., Guimarães, N. S., \& Gomes, J. M. G. (2020). Estado nutricional, fatores de risco e comorbidades em adultos portadores de diabetes mellitus tipo 2. HU Revista, 46, 1-9.

de Almeida Roediger, M., Marucci, M. D. F. N., Gobbo, L. A., Dourado, D. A. Q. S., Santos, J. L. F., de Oliveira Duarte, Y. A., \& Lebrão, M. L. Diabetes mellitus referida: incidência e determinantes, em coorte de idosos do município de São Paulo, Brasil, Estudo SABE-Saúde, Bem-Estar e Envelhecimento.

de Vargas, L. B., Santos, O. F., Magalhães, L. S., Kilpp, D. S., Bertacco, R. A., Marques, A. Y. C., \& Borges, L. R. (2021). Avaliação da qualidade da dieta de pacientes diabéticos tipo 2 atendidos no Ambulatório de Nutrição da Universidade Federal de Pelotas. Revista Da Associação Brasileira De NutriçãoRASBRAN, 12(1), 52-70.

do Nascimento Mota, T., Soares, G. P., de Araújo Gomes, A., Vieira, G. G., Campos, L. N., de Araújo Gomes, M., \& Lopes, T. V. (2020). Complicações da revascularização do miocárdio em pacientes com diabetes mellitus. Revista Eletrônica Acervo Científico, 17, e5825-e5825.

Dos Anjos, J. G., \& Leite, L. H. M. (2020). Atividades de autocuidado nutricional entre indivíduos com diabetes tipo 2. Revista Da Associação Brasileira De Nutrição-RASBRAN, 11(1), 19-34.

dos Santos, R., \& Henrique, P. Comparação do perfil antropométrico de adolescentes das redes pública e privada da cidade de Araras/SP e região.

Ely, K. Z., Spode, F., Barcella, R. C., Schonhofen, I. V., Paiva, D., Pohl, H. H., \& Possuelo, L. G. (2017). Exercício físico na diabetes mellitus, uma revisão narrativa. Cinergis, 18, 381-385.

Francisco, P. M. S. B., Rodrigues, P. S., Costa, K. S., Tavares, N. U. L., Tierling, V. L., Barros, M. B. D. A., \& Malta, D. C. (2019). Prevalência de diabetes em adultos e idosos, uso de medicamentos e fontes de obtenção: uma análise comparativa de 2012 e 2016. Revista Brasileira de Epidemiologia, 22 , e190061.

Greco-Soares, J. P., \& Dell'Aglio, D. D. (2017). Adesão ao tratamento em adolescentes com diabetes mellitus tipo 1. Psicologia, Saúde e Doenças, 18(2), $322-334$

Kuczynski, K. Z., Klipp, D. S., Brod, L., Abib, R. T., \& Borges, L. R. (2019). Avaliação do conhecimento sobre nutrição de diabéticos atendidos em um centro de referência do sul do Brasil. RBONE-Revista Brasileira De Obesidade, Nutrição E Emagrecimento, 13(83), 1151-1158.

Oliveira, J. E. P. D., \& Vencio, S. (2017). Diretrizes da Sociedade Brasileira de Diabetes 2017-2018. Editora Clannad, 91.

R Development Core Team (2021). R: A language and environment for statistical computing. R Foundation for Statistical Computing, Vienna, Austria. ISBN 3-900051-07-0. <http://www.R-project.org>

Salin, A. B., Bandeira, M. S. N., Freitas, P. R. N. D. O., \& Serpa, I. (2019). Diabetes Mellitus tipo 2: perfil populacional e fatores associados à adesão terapêutica em Unidades Básicas de Saúde em Porto Velho-RO. Revista Eletrônica Acervo Saúde, (33), e1257-e1257.

Solbiati, V. P., de Oliveira, N. R. C., Teixeira, C. V. L. S., \& Gomes, R. J. (2018). Adesão ao tratamento para prevenir agra vos relacionados à hipertensão arterial e ao diabetes. RBONE-Revista Brasileira De Obesidade, Nutrição E Emagrecimento, 12(73), 629-633. 